\title{
"Suicide CARE" (Standardized Suicide Prevention Program for Gatekeeper Intervention in Korea): An Update
}

\author{
Seon-Cheol Park ${ }^{1 *}$, Kyoung-Sae Na ${ }^{2 \star}$, Sun-Jung Kwon ${ }^{3}$, Minjae Kim ${ }^{4}$, Hyoung-Jun Kim ${ }^{5}$, Myungjae Baik ${ }^{6}$, \\ Jinmi Seol ${ }^{5}$, Eun Ji An ${ }^{7}$, Sang Min Lee ${ }^{8}$, Eun-Jin Lee ${ }^{9}$, Meerae Lim ${ }^{5}$, Sung Joon Cho' ${ }^{10}$, Gwang Hun Kim ${ }^{11}$, \\ Nari Kim ${ }^{8}$, Hong Jin Jeon ${ }^{12}$, Jong-Woo Paik ${ }^{8}$, Kang Seob Oh ${ }^{10}$, and Hwa-Young Lee ${ }^{13 凶}$ \\ ${ }^{1}$ Department of Psychiatry, Inje University Haeundae Paik Hospital, Busan, Republic of Korea \\ ${ }^{2}$ Department of Psychiatry, Gachon University Gil Medical Center, Incheon, Republic of Korea \\ ${ }^{3}$ Suicide Prevention Center, Republic of Korea Air Force, Daejeon, Republic of Korea \\ ${ }^{4}$ Department of Neuropsychiatry, Seoul National University Hospital, Seoul, Republic of Korea \\ ${ }^{5}$ Workplace Mental Health Institute, Kangbuk Samsung Hospital, Sungkyunkwan University School of Medicine, Seoul, Republic of Korea \\ ${ }^{6}$ Mental Health Center, The Armed Forces Capital Hospital, Seongnam, Republic of Korea \\ ${ }^{7}$ Department of Psychiatry, The Armed Forces Capital Hospital, Seongnam, Republic of Korea \\ ${ }^{8}$ Department of Psychiatry, Kyung Hee University School of Medicine, Seoul, Republic of Korea \\ ${ }^{9}$ Department of Social Welfare, Suwon Science College, Hwaseong, Republic of Korea \\ ${ }^{10}$ Department of Psychiatry, Kangbuk Samsung Hospital, Sungkyunkwan University School of Medicine, Seoul, Republic of Korea \\ ${ }^{11}$ Korea Association for Suicide Prevention, Seoul, Republic of Korea \\ ${ }^{12}$ Department of Psychiatry, Samsung Medical Center, Sungkyunkwan University School of Medicine, Seoul, Republic of Korea \\ ${ }^{13}$ Department of Psychiatry, Soonchunhyang University Cheonan Hospital, Cheonan, Republic of Korea
}

Objective In 2011, "Suicide CARE" (Standardized Suicide Prevention Program for Gatekeeper Intervention in Korea) was originally developed for the early detection of warning signs of suicide completion, since there is a tendency to regard emotional suppression as a virtue of Korean traditional culture. A total of 1.2 million individuals completed the training program of "Suicide CARE" in Korea.

Methods More sophisticated suicide prevention approaches according to age, sex, and occupation have been proposed, demanding for a more detailed revision of "Suicide CARE." Thus, during the period from August 2019 to February 2020, "Suicide CARE" has been updated to version 2.0. The assessments on domestic gatekeeper training programs for suicide prevention, international gatekeeper training programs for suicide prevention, psychological autopsy interview reports between 2015 and 2018, and the evaluation of feedback from people who completed "Suicide CARE" version 1.6 training were performed.

Results We describe the revision process of "Suicide CARE," revealing that "Suicide CARE" version 2.0 has been developed using an evidence-based methodology.

Conclusion It is expected that "Suicide CARE" version 2.0 be positioned as the basic framework for many developing gatekeeper training programs for suicide prevention in Korea in the near future.

Psychiatry Investig 2020;17(9):911-924

Key Words Suicide Prevention Program, Gatekeeper Intervention, “Suicide CARE,” Revision, Korea.

\section{INTRODUCTION}

Suicide is the most important public mental health issue in Korea. ${ }^{1-4}$ From 2003 to 2016, Korea has reported the highest

Received: May 7, 2020 Accepted: June 29, 2020

$\bowtie$ Correspondence: Hwa-Young Lee, MD, PhD

Department of Psychiatry, Soonchunhyang University Cheonan Hospital, 31 Suncheonhyang 6-gil, Dongnam-gu, Cheonan 31151, Republic of Korea Tel: +82-41-570-2280, Fax: +82-41-592-3810, E-mail: leehway@gmail.com

*These authors contributed equally to this work.

(a) This is an Open Access article distributed under the terms of the Creative Commons Attribution Non-Commercial License (https://creativecommons.org/licenses/bync/4.0) which permits unrestricted non-commercial use, distribution, and reproduction in any medium, provided the original work is properly cited. suicide rate among the Organization for Economic Cooperation and Development (OECD) countries. Since Lithuania joined the OECD in 2017, the highest suicide rate among the OECD countries, in just a year, was reported by Lithuania. Thus, Korea reported the second-highest suicide rate among the OECD countries. However, the statistics for suicide have remained troubling, as follows: As of 2018, the death rate due to suicide per 100,000 persons was 26.6 in Korea, which was much higher than the average suicide death rate of 11.6 in the other OECD countries. ${ }^{5}$ Recently, the "National Suicide Prevention Action Plan" of Ministry of Health and Welfare of Korea has aimed to reduce suicide death rate to less than 
20 per 100,000 persons by 2022 and total completed suicides to less than 10,000 persons per year. ${ }^{6}$ A recent systematic review reported that restricting access to lethal means and conducting school-based awareness programs were sufficiently evidenced to prevent suicide. In addition, effective pharmacological and psychological treatments for depression, gatekeeper training, education of physicians, and internet and helpline support have been proposed as evidence-based suicide prevention strategies. However, screening in primary care and general public education and media guidelines are insufficiently evidenced in the prevention of suicide. ${ }^{7}$ Most importantly, gatekeeper training has been considered to be an effective suicide prevention strategy for young people by the Center for Disease Control and Prevention. In terms of gatekeeper training programs for suicide prevention, social workers, caregivers, and churchmen should identify people with a high risk of suicidality and connect them with psychosocial support systems. ${ }^{8}$

In Korea, evidence-based suicide prevention strategies have been developed as follows: The first suicide prevention program for the members of the Korean Medical Association was held in 2010. In addition, in terms of gatekeeper training, the Standardized Suicide Prevention Program for Gatekeeper Intervention in Korea was originally developed by the Korea Association for Suicide Prevention, under the support of the Life Insurance Philanthropy Foundation in 2011. ${ }^{9}$ Thus, "Suicide CARE" was developed for the early detection of danger signals of suicide completion, since there is a tendency to regard emotional suppression as a virtue of Korean traditional culture. The gatekeeper training program has been popularly referred to as "Bo-Deud-Mal (Bogo Deudgo Malhagi)" in Korean and translated to "Suicide CARE" ("Careful observation," "Active listening," and "Risk evaluation and Expert referral") in English. "Suicide CARE" provides specific guidelines regarding gatekeeper intervention for people with a high risk of suicide. In addition, the gatekeeper training program has been divided into three parts according to the name as follows: "Careful observation" covers the detection of verbal and non-verbal signals for suicidal intents. "Active listening" aims to actively hear the cause of suicidal intention, and "Risk evaluation and Expert referral " involves referring suicidal persons to psychiatric professionals. After the first demonstration of "Suicide CARE" in January 2013, an executive committee, which consisted of its developing team, was established by the Korea Suicide Prevention Center. Based on the principle that any individual could complete the program free of charge, education was mainly provided through community mental health welfare centers nationwide. The instructors were limited to mental health professionals with more than two years of suicide prevention work experience, and they completed a 2-day course of "Suicide CARE" with faculty supervision. Instructors were provided with instructor manuals with detailed information on contents, and gatekeepers were provided with the contents of workbooks, video clips, and role plays. It was designed to simulate the experience of the activity. The certificates of completion for "Suicide CARE" were downloaded through the website of the Korea Suicide Prevention Center (adapted from http://www.spckorea.or.kr/index.php). By 2019, 1.2 million individuals had completed life-saving education. ${ }^{10}$

In addition, the gatekeeper training program was revised to provide individually differentiated programs focusing on specific groups, including young people, office workers, and the army, navy, and air forces, under the support of the Ministry of Health and Welfare, in 2014. The revised version number of the gatekeeper training program was marked as 1.6 (not 2.0) because the revisions made were modest. "Suicide CARE" version 1.6 consists of a workbook, transcript of the lecture, and video clips to improve gatekeeper training. ${ }^{11}$ As shown in Figure 1, each part named "Careful observation," "Active listening," and "Risk evaluation and Expert referral" is represented by an individual image, in "Suicide CARE" versions 1.0 and 1.6, respectively. 9,11 A telephone survey of 800 people who had completed the educational course of the program in 2013 re-

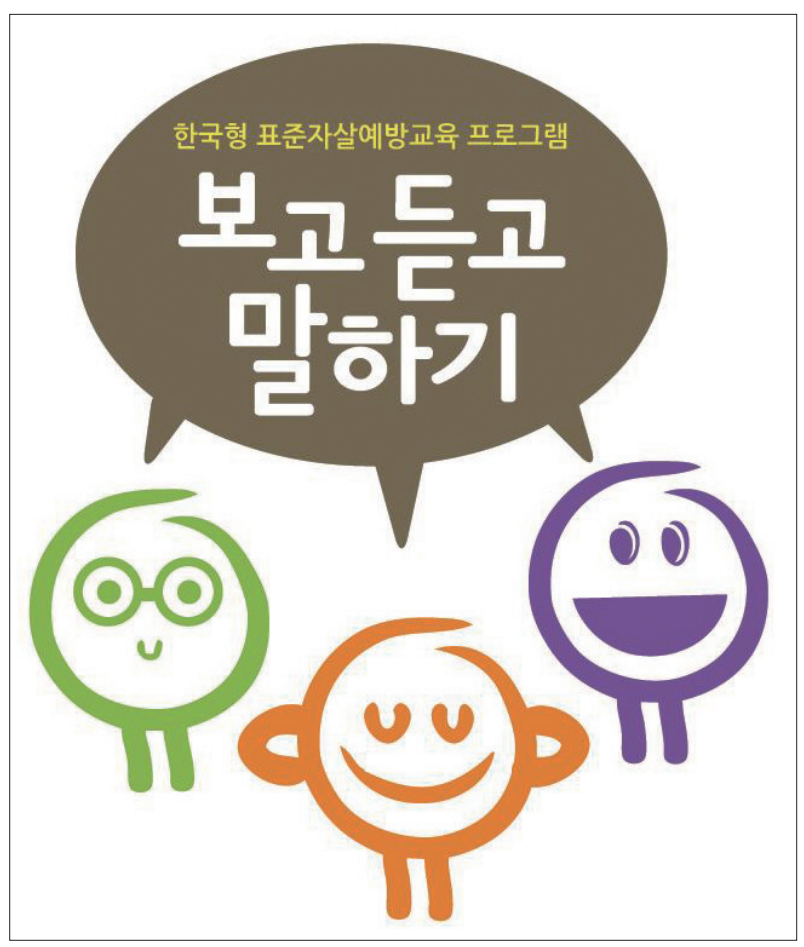

Figure 1. Image representing "Careful observation," "Active listening," and "Risk evaluation and Expert referral" in "Suicide CARE" versions 1.0 and 1.6. Adapted from "Suicide CARE" (Standardized Suicide Prevention Program for Gatekeeper Intervention in Korea) version 1.5 Workbook. Seoul: Korea Association for Suicide Prevention \& Korea Suicide Prevention Center, 2014, according to the Creative Commons license. ${ }^{9,13}$ From left to right, characterized images symbolize the "Careful observation," "Active listening," and "Risk evaluation and Expert referral" parts. 
ported that the gatekeeper's intervention for suicide prevention could be favorably supported by "Suicide CARE" version 1.6. ${ }^{10}$ Owing to the considerable amount of time since its initial creation, it was required that "Suicide CARE" should be revised entirely. It is also necessary that the revised gatekeeper training program should be underpinned by current study findings and empirical knowledge. In addition, the gatekeeper training program must be revised based on psychological autopsy interview findings ${ }^{12}$ in the most recent 5 years in Korea. Since suicide has been the most common cause of death among those in their 10s, 20s, and 30s, and the second most common among those in their 40s to 50s in Korea, it has been presumed that the development of age-group-based differentiated suicide prevention approaches is urgently needed. Thus, "Suicide CARE" was revised from version 1.6 to 2.0, between August 2019 and February 2020, by the multidisciplinary team including psychiatrists, psychologists, and social workers. The revision has been mainly based on the numerous domestic and international gatekeeper training programs for suicide prevention and the recent psychological autopsy findings of Korea. Therefore, in this paper, we aim to present the detailed revision process of "Suicide CARE" from version 1.6 to 2.0.

\section{METHODS}

Under the support of Korea Suicide Prevention Center, which is a designated agency of the Ministry of Health and Welfare, during the period from August 2019 to March 2020, "Suicide CARE" has been revised from version 1.6 to 2.0. The revision process consisted of: 1) reviews about domestic gatekeeper training programs for suicide prevention, 2) reviews about international gatekeeper training programs for suicide prevention, 3) reviews about psychological autopsy interview reports, 2018, and 4) reviews about feedback from persons who completed "Suicide CARE" version 1.6.

\section{RESULTS}

\section{Domestic gatekeeper training programs}

As shown in Table 1, the Korea Suicide Prevention Center has managed a registration system for domestic gatekeeper training programs to introduce evidence-based suicide prevention interventions. ${ }^{13}$ In addition, the Korea Suicide Prevention Center has certified the evidence levels of domestic gatekeeper training programs. Thus, 68 different programs have been certified. The certification criteria are classified into SECTION 1, SECTION 2, and SECTION 3 as follows: SECTION 1 denotes the suicide prevention intervention in which the content and efficacy are evidenced by a structured empirical study (randomized controlled study or non-randomized controlled study). SECTION 2 denotes expert consensus-based interventions or recommendations in the general setting. SECTION 3 denotes interventions or recommendations in special settings (i.e., public awareness and promotion programs, education or training programs, protocols or guidelines, and screening tools). A review of the current domestic gatekeeper training programs reveals the following limitations: First, as shown in Table 1, most of the domestic gatekeeper training programs were certified as low-level. Among 68 different programs, $89.7 \%(\mathrm{n}=61)$ were defined as SECTION 3, whereas $8.8 \%(\mathrm{n}=6)$ were defined as SECTION 2. Only $1.5 \%(\mathrm{n}=1)$ of the programs was defined as SECTION 1, according to the criteria of the Korea Suicide Prevention Center. ${ }^{16}$ Second, it has been speculated that the domestic gatekeeper training programs for suicide prevention are in a state of contention with many differing views. In addition, more than half of the programs were developed by institutions located in the National Capital region (i.e., Seoul, Incheon, Gyeonggi-do). Most of the domestic gatekeeper training programs are available only for targeted regional residents and not for non-targeted regional residents. Thus, to overcome these limitations, it has been suggested that a new domestic gatekeeper training program with a high level of evidence and a national range of availability should be developed. Most programs commonly focus on enhancing gatekeepers' understanding of the risk and protective factors of suicide to connect highrisk suicidal persons with mental professionals early. In addition, all programs are free of charge. Herein, it has been proposed that the revised version of "Suicide CARE" should be supported by high-level evidence available nation-wide and focused on gatekeepers' early detection and management of suicidal risk.

\section{International gatekeeper training programs}

Suicide Prevention Resource Center (SPRC), Resources and Programs (available from: http:// http://www.sprc.org/resources-programs $)^{78}$ were searched from inception until October 30th, 2019. As shown in Table 2, we reviewed the international government-initiated suicide prevention programs, including the Office Worker Suicide Prevention Policy (World Health Organization), data from the Bureau of Labor in Quebec (Canada), Montreal police officer's Together for Life (Canada), suicide prevention model for office workers (Canadian Association for Suicide Prevention), Victorian Work-Related Fatality Database (VWRFD) (Australia), MATES in Construction: construction worker suicide prevention (Australia), and others. In addition, we reviewed the international private corporate-initiated suicide prevention programs, including the Applied Suicide Intervention Skills Training (ASIST), Question, Persuade, Refer (QPR), Working Minds, and others. We extracted common factors, characteristics, 
Table 1. Domestic gatekeeper training programs for suicide prevention

\begin{tabular}{|c|c|c|c|}
\hline Program & $\begin{array}{l}\text { Development } \\
\text { institution }\end{array}$ & Highlight & SECTION \\
\hline $\begin{array}{l}\text { Suicide Reporting Recommendation } \\
\text { Guideline } 3.0^{14}\end{array}$ & $\begin{array}{l}\text { Korea Suicide } \\
\text { Prevention Center }\end{array}$ & Principles of suicide-related reporting & 2 \\
\hline $\begin{array}{l}\text { Suicide Prevention Education Program } \\
\text { for Senior Service Provider }{ }^{15}\end{array}$ & $\begin{array}{l}\text { Korea Suicide } \\
\text { Prevention Center }\end{array}$ & $\begin{array}{l}\text { Educate senior service providers on the characteristics of } \\
\text { elderly and risk factors of elderly suicide }\end{array}$ & 3 \\
\hline $\begin{array}{l}\text { Cognitive Behavioral Program for } \\
\text { Suicidal Attempter }{ }^{16}\end{array}$ & $\begin{array}{l}\text { Korea Suicide } \\
\text { Prevention Center }\end{array}$ & $\begin{array}{l}\text { Understand cognitive distortion, automatic thoughts, and } \\
\text { emotional response and behavior of suicide attempters, } \\
\text { education on cognitive behavioral programs for prevention } \\
\text { of suicidal reattempts }\end{array}$ & 3 \\
\hline 'Suicide CARE' for Air Force ${ }^{17}$ & $\begin{array}{l}\text { Korea Suicide } \\
\text { Prevention Center }\end{array}$ & $\begin{array}{l}\text { Suicide prevention education program for the air force, } \\
\text { understanding of suicide, and training gatekeepers of air force }\end{array}$ & 3 \\
\hline $\begin{array}{l}\text { Senior Suicide Prevention } \\
\text { Education Program }\end{array}$ & $\begin{array}{l}\text { Korea Suicide } \\
\text { Prevention Center }\end{array}$ & $\begin{array}{l}\text { Understand geriatric depression and suicide, Improve } \\
\text { awareness on senior suicide prevention }\end{array}$ & 3 \\
\hline $\begin{array}{l}\text { Suicide Prevention Education } \\
\text { Program for Office Worker }{ }^{19}\end{array}$ & $\begin{array}{l}\text { Korea Suicide } \\
\text { Prevention Center }\end{array}$ & $\begin{array}{l}\text { Understand the risk factors of suicide, self-assessment, and } \\
\text { suicidal signs, conduct suicide prevention interventions }\end{array}$ & 3 \\
\hline $\begin{array}{l}\text { Suicide Prevention Education for } \\
\text { Emergency Medical Worker }{ }^{20}\end{array}$ & $\begin{array}{l}\text { Korea Suicide } \\
\text { Prevention Center }\end{array}$ & $\begin{array}{l}\text { Understand psychiatric assessment and interview principles } \\
\text { of suicide, introduce suicide screening tools and } \\
\text { suicide-related pharmacotherapy and physical restraints, } \\
\text { conduct suicide-related emergency medical services }\end{array}$ & 3 \\
\hline $\begin{array}{l}\text { Suicide Prevention Gatekeeper } \\
\text { Education Program }{ }^{21}\end{array}$ & $\begin{array}{l}\text { Korea Suicide } \\
\text { Prevention Center }\end{array}$ & $\begin{array}{l}\text { Understand high-risk suicidal groups, detect high-risk } \\
\text { suicidal groups, educate helping skills of gatekeepers }\end{array}$ & 3 \\
\hline $\begin{array}{l}\text { Senior Suicide Prevention } \\
\text { Gatekeeper Education Program }\end{array}$ & $\begin{array}{l}\text { Korea Suicide } \\
\text { Prevention Center }\end{array}$ & $\begin{array}{l}\text { Educate on suicide prevention for the elderly, } \\
\text { train gatekeepers, enhance the mental health of the elderly }\end{array}$ & 3 \\
\hline $\begin{array}{l}\text { Crisis Management Protocol for } \\
\text { Suicide and Mental Disorder }{ }^{23}\end{array}$ & $\begin{array}{l}\text { Seoul Suicide } \\
\text { Prevention Center }\end{array}$ & $\begin{array}{l}\text { Integrated crisis management service system, continuous } \\
\text { management service system for mental hospitals and } \\
\text { community mental health welfare centers }\end{array}$ & 2 \\
\hline $\begin{array}{l}\text { Suicide Crisis Intervention } \\
\text { Handbook }^{24}\end{array}$ & $\begin{array}{l}\text { Seoul Suicide } \\
\text { Prevention Center }\end{array}$ & $\begin{array}{l}\text { Systemize the intervention strategies through understanding } \\
\text { suicide, education on suicidal risk assessment, 6-step } \\
\text { counseling and intervention skills }\end{array}$ & 3 \\
\hline $\begin{array}{l}\text { Suicide Prevention Gatekeeper } \\
\text { Training Program } \\
25\end{array}$ & $\begin{array}{l}\text { Seoul Suicide } \\
\text { Prevention Center }\end{array}$ & $\begin{array}{l}\text { Understand suicide, establish strategies for suicide } \\
\text { prevention, gatekeeper work activity }\end{array}$ & 3 \\
\hline $\begin{array}{l}\text { Manual for Counseling and Self-Help } \\
\text { Group for Survivor of Bereavement } \\
\text { by Suicide }\end{array}$ & $\begin{array}{l}\text { Seoul Suicide } \\
\text { Prevention Center }\end{array}$ & $\begin{array}{l}\text { Understand counseling and self-help groups for bereaved } \\
\text { survivors of suicide }\end{array}$ & 2 \\
\hline $\begin{array}{l}\text { Suicide Prevention Education for } \\
\text { Emergency Worker }{ }^{27}\end{array}$ & $\begin{array}{l}\text { Seoul Suicide } \\
\text { Prevention Center }\end{array}$ & $\begin{array}{l}\text { Enhance gatekeepers' intervention skills for high suicidality, } \\
\text { control the quality of community-based suicide prevention } \\
\text { services }\end{array}$ & 3 \\
\hline $\begin{array}{l}\text { Suicide Prevention Professional } \\
\text { Training Program } \\
28\end{array}$ & $\begin{array}{l}\text { Seoul Suicide } \\
\text { Prevention Center }\end{array}$ & $\begin{array}{l}\text { Understand skill, application, and supervision of motivation } \\
\text { enhancement interviews }\end{array}$ & 3 \\
\hline $\begin{array}{l}\text { Gatekeeper Training Program for } \\
\text { Teacher }^{29}\end{array}$ & $\begin{array}{l}\text { Seoul Suicide } \\
\text { Prevention Center }\end{array}$ & Train teachers on suicide risk assessment of adolescents & 3 \\
\hline $\begin{array}{l}\text { Adolescent Suicide Crisis Intervention } \\
\text { Manual for Teacher and Official }{ }^{30}\end{array}$ & $\begin{array}{l}\text { Incheon Suicide } \\
\text { Prevention Center }\end{array}$ & $\begin{array}{l}\text { Be aware of problem of adolescent suicide, prevent adolescent } \\
\text { suicide, provide for adolescents with suicidality, } \\
\text { interconnection of adolescent-related institutions }\end{array}$ & 3 \\
\hline $\begin{array}{l}\text { School-Based Suicide Postvention } \\
\text { Program }\end{array}$ & $\begin{array}{l}\text { Seoul Mental Health } \\
\text { Welfare Center }\end{array}$ & $\begin{array}{l}\text { Understand the psychological conflicts about school-related } \\
\text { suicide }\end{array}$ & 3 \\
\hline $\begin{array}{l}\text { Suicide Prevention Program of } \\
\text { Community Resident Participation }\end{array}$ & $\begin{array}{l}\text { Gangseo-gu } \\
\text { Mental Health } \\
\text { Welfare Center, Seoul }\end{array}$ & $\begin{array}{l}\text { Detect suicide problems within the community, establish } \\
\text { leadership, increase community resident suicide prevention } \\
\text { activities }\end{array}$ & 3 \\
\hline
\end{tabular}


Table 1. Domestic gatekeeper training programs for suicide prevention (continued)

\begin{tabular}{|c|c|c|c|}
\hline Program & $\begin{array}{l}\text { Development } \\
\text { institution }\end{array}$ & Highlight & SECTION \\
\hline $\begin{array}{l}\text { Mental Health Promotion and } \\
\text { Happiness Enhancement } \\
\text { Thank You Program } \\
\text { 33 }\end{array}$ & $\begin{array}{l}\text { Seongdong-gu } \\
\text { Mental Health } \\
\text { Welfare Center, Seoul }\end{array}$ & $\begin{array}{l}\text { Through increasing positive emotions, decrease } \\
\text { suicidality of psychiatric patients with suicidal ideation, } \\
\text { suicidal attempters, and bereaved survivors of suicide }\end{array}$ & 3 \\
\hline 'Mind Cane' ${ }^{34}$ & $\begin{array}{l}\text { Busan Mental Health } \\
\text { Welfare Center }\end{array}$ & $\begin{array}{l}\text { 10-session cognitive behavioral therapy for elderly } \\
\text { with depressive mood and suicidal ideation }\end{array}$ & 3 \\
\hline $\begin{array}{l}\text { Psychological Emotion Support } \\
\text { Recommendation for Official } \\
\text { Whose Client Commit Suicide }{ }^{35}\end{array}$ & $\begin{array}{l}\text { Busan Mental Health } \\
\text { Welfare Center }\end{array}$ & $\begin{array}{l}\text { Recommend institution response (i.e., situation report, } \\
\text { administrative management) and psychological } \\
\text { emotional support (i.e., case supervision, work } \\
\text { cooperation, vacation support, counseling support for } \\
\text { psychological conflicts) }\end{array}$ & 2 \\
\hline $\begin{array}{l}\text { Psychological Support for Survivor } \\
\text { of Bereavement by Suicide }{ }^{36}\end{array}$ & $\begin{array}{l}\text { Incheon Mental } \\
\text { Health Welfare Center }\end{array}$ & Understand bereaved survivors of suicide & 3 \\
\hline $\begin{array}{l}\text { Senior Suicidal Crisis Management } \\
\text { Manual }^{37}\end{array}$ & $\begin{array}{l}\text { Incheon Mental } \\
\text { Health Welfare Center }\end{array}$ & $\begin{array}{l}\text { Understand suicide by elderly and interventions in } \\
\text { actual practice }\end{array}$ & 3 \\
\hline $\begin{array}{l}\text { 'Suicide Prevention and Crisis } \\
\text { Intervention Manual' for Suicide } \\
\text { Prevention Official }^{38}\end{array}$ & $\begin{array}{l}\text { Incheon Mental } \\
\text { Health Welfare Center }\end{array}$ & $\begin{array}{l}\text { Understand suicide, crisis management, emergency } \\
\text { intervention principle, and case management }\end{array}$ & 2 \\
\hline Adolescent Life Respect Education ${ }^{39}$ & $\begin{array}{l}\text { Daegu Mental Health } \\
\text { Welfare Center }\end{array}$ & $\begin{array}{l}\text { Prevent adolescent suicide, educate intervention skill } \\
\text { for suicide }\end{array}$ & 3 \\
\hline $\begin{array}{l}\text { Senior Life Respect Education } \\
\text { Program, 'Life Charger' }\end{array}$ & $\begin{array}{l}\text { Daegu Mental Health } \\
\text { Welfare Center }\end{array}$ & $\begin{array}{l}\text { Understand the definition of life chargers, detect suicidal } \\
\text { ambivalence and signs }\end{array}$ & 3 \\
\hline $\begin{array}{l}\text { Crisis Intervention Program of } \\
\text { Gwangju }^{41}\end{array}$ & $\begin{array}{l}\text { Gwangju Mental } \\
\text { Health Welfare Center }\end{array}$ & $\begin{array}{l}\text { Detect suicidal crisis signs, increase interviewing skills } \\
\text { of suicide prevention officials }\end{array}$ & 3 \\
\hline 'Life Zone' ${ }^{\prime 2}$ & $\begin{array}{l}\text { Buk-gu Mental Health } \\
\text { Welfare Center, Gwangju }\end{array}$ & $\begin{array}{l}\text { Structured education program for instructor training, } \\
\text { train gatekeepers to help high-risk suicidal groups }\end{array}$ & 3 \\
\hline 'Mind Friends' ${ }^{33}$ & $\begin{array}{l}\text { Buk-gu Mental Health } \\
\text { Welfare Center, Gwangju }\end{array}$ & $\begin{array}{l}\text { Understand the protective and risk factors of adolescent } \\
\text { suicide and depressive symptoms }\end{array}$ & 3 \\
\hline 'What's Up?'44 & $\begin{array}{l}\text { Dong-gu Mental Health } \\
\text { Welfare Center, Gwangju }\end{array}$ & $\begin{array}{l}\text { Understand the suicidal risk of adolescents, understand } \\
\text { suicidal signs }\end{array}$ & 3 \\
\hline $\begin{array}{l}\text { Group Program Manual for Survivor } \\
\text { of Bereavement by Suicide }{ }^{45}\end{array}$ & $\begin{array}{l}\text { Gyeonggi-do Mental } \\
\text { Health Welfare Center }\end{array}$ & $\begin{array}{l}\text { Systemize standardized service of a group program for } \\
\text { the bereaved survivors of suicide, facilitate a group of } \\
\text { the survivors of suicide in the community, provide an } \\
\text { education program for grief }\end{array}$ & 3 \\
\hline Life Loving Teen Teen Class $3.0^{46}$ & $\begin{array}{l}\text { Gyeonggi-do Mental } \\
\text { Health Welfare Center }\end{array}$ & $\begin{array}{l}\text { Understand rational ideas about suicide, help an } \\
\text { adolescent friend with suicide ideation }\end{array}$ & 1 \\
\hline $\begin{array}{l}\text { Life Loving Teen Teen Class } \\
\text { Gatekeeper }^{47}\end{array}$ & $\begin{array}{l}\text { Gyeonggi-do Mental } \\
\text { Health Welfare Center }\end{array}$ & Understand life-loving and the value of life & 3 \\
\hline $\begin{array}{l}\text { Student Suicide Prevention } \\
\text { Intervention Program, 'M-love }{ }^{38}\end{array}$ & $\begin{array}{l}\text { Gyeonggi-do Mental } \\
\text { Health Welfare Center }\end{array}$ & $\begin{array}{l}\text { Provide an opportunity of emotional expression, return } \\
\text { to ordinary life through a normal grief reaction }\end{array}$ & 3 \\
\hline $\begin{array}{l}\text { Gold Medal Case Management } \\
\text { Program }^{49}\end{array}$ & $\begin{array}{l}\text { Suwon Mental Health } \\
\text { Welfare Center, } \\
\text { Gyeonggi-do }\end{array}$ & $\begin{array}{l}\text { Divide and construct goal behaviors into gold, silver, } \\
\text { and bronze medals, based on conditioning theory }\end{array}$ & 3 \\
\hline $\begin{array}{l}\text { Adolescent Suicide Prevention } \\
\text { Gatekeeper Training Program, } \\
\text { 'Between Friends' }{ }^{\text {'0 }}\end{array}$ & $\begin{array}{l}\text { Suwon Mental Health } \\
\text { Welfare Center, } \\
\text { Gyeonggi-do }\end{array}$ & $\begin{array}{l}\text { Understand the factors influencing suicidal ideation, } \\
\text { suicidal signs, protective factors against suicide, and } \\
\text { helping methods for friend }\end{array}$ & 3 \\
\hline Life Respect Manual ${ }^{51}$ & $\begin{array}{l}\text { Seongnam Mental } \\
\text { Health Welfare Center, } \\
\text { Gyeonggi-do }\end{array}$ & $\begin{array}{l}\text { Problem-solving manual for persons with suicidal } \\
\text { ideation }\end{array}$ & 3 \\
\hline
\end{tabular}


Table 1. Domestic gatekeeper training programs for suicide prevention (continued)

\begin{tabular}{|c|c|c|c|}
\hline Program & $\begin{array}{l}\text { Development } \\
\text { institution }\end{array}$ & Highlight & SECTION \\
\hline $\begin{array}{l}\text { Suicide Prevention Gatekeeper } \\
\text { Education Program } \\
52\end{array}$ & $\begin{array}{l}\text { Seongnam Mental Health } \\
\text { Welfare Center, Gyeonggi-do }\end{array}$ & $\begin{array}{l}\text { Develop a suicide prevention gatekeeper education for } \\
\text { citizens of Seongnam }\end{array}$ & 3 \\
\hline $\begin{array}{l}\text { Support Guidebook for Survivor of } \\
\text { Bereavement by Suicide }\end{array}$ & $\begin{array}{l}\text { Seongnam Mental Health } \\
\text { Welfare Center, Gyeonggi-do }\end{array}$ & $\begin{array}{l}\text { Understand community-based support services for } \\
\text { bereaved survivors of suicide }\end{array}$ & 3 \\
\hline Life Respect Green Village $e^{54}$ & $\begin{array}{l}\text { Hwaseong Mental Health } \\
\text { Welfare Center, Gyeonggi-do }\end{array}$ & Equip a safe deposit box of agricultural chemicals & 3 \\
\hline $\begin{array}{l}\text { Gatekeeper Training Program for } \\
\text { Police Officer }\end{array}$ & $\begin{array}{l}\text { Chuungcheongbuk-do } \\
\text { Mental Health Welfare Center }\end{array}$ & Recognize statistical data of police officer suicide & 3 \\
\hline $\begin{array}{l}\text { Gatekeeper Training Program for } \\
\text { Firefighter }^{56}\end{array}$ & $\begin{array}{l}\text { Chuungcheongbuk-do } \\
\text { Mental Health Welfare Center }\end{array}$ & Recognize statistical data of firefighter suicide & 3 \\
\hline Emergency Kit Emergency Box ${ }^{57}$ & $\begin{array}{l}\text { Chungchengbuk-do } \\
\text { Mental Health Welfare Center }\end{array}$ & $\begin{array}{l}\text { 1. Emergency kit (a tool to cope with physical } \\
\text { emergency situations) } \\
\text { 2. Emergency box (suicidal risk assessment, crisis } \\
\text { intervention, refer to medical institutions, and others) }\end{array}$ & 3 \\
\hline 'Suicide Crisis Management Manual'58 & $\begin{array}{l}\text { Chungcheongbuk-do } \\
\text { Mental Health Welfare Center }\end{array}$ & $\begin{array}{l}\text { Suicide crisis management manual for } 112,119 \text {, and } \\
\text { community mental health welfare centers }\end{array}$ & 2 \\
\hline Life Savor Training Program ${ }^{59}$ & $\begin{array}{l}\text { Chuungcheongbuk-do } \\
\text { Mental Health Welfare Center }\end{array}$ & Understand suicide and suicide prevention & 3 \\
\hline $\begin{array}{l}\text { Chungbuk Stop of Suicide (SOS) } \\
\text { Project }^{60}\end{array}$ & $\begin{array}{l}\text { Chungcheongbuk-do } \\
\text { Mental Health Welfare Center }\end{array}$ & $\begin{array}{l}\text { Suicidal crisis intervention program for policemen, } \\
\text { firefighters, and mental health welfare center officials }\end{array}$ & 3 \\
\hline Running Life ${ }^{61}$ & $\begin{array}{l}\text { Chungcheongnam-do } \\
\text { Mental Health Welfare Center }\end{array}$ & $\begin{array}{l}\text { Suicide prevention education for adolescent, adults, } \\
\text { and seniors }\end{array}$ & 3 \\
\hline $\begin{array}{l}\text { Motivation Enhancement Cognitive } \\
\text { Behavioral Program 2.0, 'Empathy } 3^{\prime 62}\end{array}$ & $\begin{array}{l}\text { Gumi Mental Health Welfare } \\
\text { Center, Gyeongsangbuk-do }\end{array}$ & $\begin{array}{l}\text { Understand suicidal intent, enhance life motivation, } \\
\text { construct a safety net }\end{array}$ & 3 \\
\hline $\begin{array}{l}\text { Short-term Intervention Program 2.0, } \\
\text { 'Empathy } 1^{\prime 63}\end{array}$ & $\begin{array}{l}\text { Gumi Mental Health Welfare } \\
\text { Center, Gyeongsangbuk-do }\end{array}$ & $\begin{array}{l}\text { A short-term intervention program for suicide } \\
\text { attempters and high-risk suicidal groups }\end{array}$ & 3 \\
\hline Peer Life Savor Training Program ${ }^{64}$ & $\begin{array}{l}\text { Gumi Mental Health Welfare } \\
\text { Center, Gyeongsangbuk-do }\end{array}$ & $\begin{array}{l}\text { Educate on the four phases including irrational } \\
\text { belief phase, rational belief construction phase, } \\
\text { rational behavior construction phase, and rational } \\
\text { behavior acquirement phase }\end{array}$ & 3 \\
\hline $\begin{array}{l}\text { Life Loving Gatekeeper Training } \\
\text { Program and Instructor Training } \\
\text { Program }^{65}\end{array}$ & $\begin{array}{l}\text { Jeollabuk-do Mental Health } \\
\text { Welfare Center }\end{array}$ & $\begin{array}{l}\text { Increase understanding of suicide, detect suicidal } \\
\text { signs, educate on interview skills and attitude and } \\
\text { assessment and evaluation of crisis situations }\end{array}$ & 3 \\
\hline Suicide Prevention Program ${ }^{66}$ & $\begin{array}{l}\text { Jeollabuk-do Mental Health } \\
\text { Welfare Center }\end{array}$ & $\begin{array}{l}\text { Improve understanding of suicide for Jeollabuk-do } \\
\text { people, reduce the suicide rate in Jeollabuk-do }\end{array}$ & 3 \\
\hline $\begin{array}{l}\text { Life Loving Gatekeeper Professional } \\
\text { Instructor Program } \\
67\end{array}$ & $\begin{array}{l}\text { Jeollabuk-do Mental Health } \\
\text { Welfare Center }\end{array}$ & $\begin{array}{l}\text { Understand suicide and theory about suicide, } \\
\text { educate on suicidal risk assessments }\end{array}$ & 3 \\
\hline 'Knocking Repeatedly'68 & $\begin{array}{l}\text { Wanju Mental Health Welfare } \\
\text { Center, Jeollabuk-do }\end{array}$ & $\begin{array}{l}\text { Reduce suicide ideation and depressive mood of } \\
\text { high-risk suicide groups }\end{array}$ & 3 \\
\hline 'Hello?'69 & $\begin{array}{l}\text { Jeollanam-do Mental Health } \\
\text { Welfare Center }\end{array}$ & $\begin{array}{l}\text { Understand suicide, conduct early detect and early } \\
\text { management of high-risk suicidal groups, } \\
\text { decrease suicidal rate through fostering a life } \\
\text { respecting culture }\end{array}$ & 3 \\
\hline $\begin{array}{l}\text { Life Loving Gatekeeper Training } \\
\text { Program }^{70}\end{array}$ & $\begin{array}{l}\text { Jeju-do Mental Health Welfare } \\
\text { Center }\end{array}$ & $\begin{array}{l}\text { Understand the characteristics of elderly suicides, } \\
\text { detect and help high-risk suicide groups }\end{array}$ & 3 \\
\hline $\begin{array}{l}\text { Gatekeeper Education Program } \\
\text { for Police Officer }\end{array}$ & Korea National Police Agency & $\begin{array}{l}\text { Recognize suicidal risk of police officers, improve } \\
\text { the connection with resources which provide } \\
\text { appropriate help }\end{array}$ & 3 \\
\hline
\end{tabular}


Table 1. Domestic gatekeeper training programs for suicide prevention (continued)

\begin{tabular}{|c|c|c|c|}
\hline Program & $\begin{array}{l}\text { Development } \\
\text { institution }\end{array}$ & Highlight & SECTION \\
\hline Suicidality Screening Program ${ }^{72}$ & $\begin{array}{l}\text { Department of Epidemiology, } \\
\text { The Catholic University of Korea, } \\
\text { Seoul }\end{array}$ & $\begin{array}{l}\text { Conduct community-based suicidality screening } \\
\text { tests and refer persons with high-risk suicidality } \\
\text { to mental health institutions }\end{array}$ & 3 \\
\hline TLC Academy, 'Life Loving ${ }^{73}$ & $\begin{array}{l}\text { Nazarene University, Cheonan, } \\
\text { Chungcheongnam-do }\end{array}$ & $\begin{array}{l}\text { Understand suicide and suicide prevention } \\
\text { programs, train counseling, conduct a suicide } \\
\text { prevention campaign }\end{array}$ & 3 \\
\hline Life Saver Education Book ${ }^{74}$ & $\begin{array}{l}\text { Life Respect Education } \\
\text { Association, Seoul }\end{array}$ & $\begin{array}{l}\text { Educate psychology to respect life, educate suicide } \\
\text { prevention for respect of life }\end{array}$ & 3 \\
\hline $\begin{array}{l}\text { Adolescent Suicide Prevention } \\
\text { Education Program, 'I Love You'75 }\end{array}$ & $\begin{array}{l}\text { Life Line Korea, Samsung Life } \\
\text { Insurance, Community } \\
\text { Chest of Korea, Korea Health } \\
\text { Promotion Institute }\end{array}$ & $\begin{array}{l}\text { Educate intervention skill for adolescents with } \\
\text { suicidal crises, educate school-based suicide } \\
\text { prevention intervention }\end{array}$ & 3 \\
\hline $\begin{array}{l}\text { Life Loving Gatekeeper Basic } \\
\text { Education Program }{ }^{76}\end{array}$ & $\begin{array}{l}\text { Life Hope Protestant Suicide } \\
\text { Prevention Center, Seoul }\end{array}$ & $\begin{array}{l}\text { Suicide prevention intervention program for } \\
\text { adolescents }\end{array}$ & 3 \\
\hline $\begin{array}{l}\text { Sexual Minority Suicide Prevention } \\
\text { Gatekeeper Training Program }\end{array}$ & $\begin{array}{l}\text { Korean Gay Humanity } \\
\text { Movement Organization }\end{array}$ & $\begin{array}{l}\text { Understand suicidal signs and suicidal ideation of } \\
\text { sexual minorities, improve self-help of gatekeepers }\end{array}$ & 3 \\
\hline
\end{tabular}

and availability in Korean contexts from the international gatekeeper training programs. Reviews of the international gatekeeper training programs presented major considerations as follows: First, each of the international gatekeeper training programs has been developed to be used consistently with each of the special conditions for the countries. It has been concluded that most international gatekeeper training programs may be inconsistent with the particular conditions of suicide in Korea. Second, it has been assumed that the early detection of suicide risk signals should be an essential content of "Suicide CARE" version 2.0. Thus, the "Careful observation" part has been proposed as the most important portion in the revision process of "Suicide CARE" from version 1.6 to 2.0. Third, since most of the international gatekeeper training programs require a range of tuition fees, it is speculated that the fees can contribute to the main obstacles for educating and training gatekeepers. Thus, it was concluded that "Suicide CARE" should be distributed free of charge. Fourth, role play and group simulation are regarded as the main sections in many of the international gatekeeper training programs. Thus, it has been proposed that role-play or group simulation should be included in "Suicide CARE" version 2.0.

\section{Psychological autopsy interview reports 2015-2018}

The Korea Psychological Autopsy Center has published psychological autopsy interview reports, which present the clinical characteristics of 391 Korean suicide completers from 2015 to 2018, based on interviews with family survivors. ${ }^{12}$ The contents for the psychological autopsy interview reports, 2015-2018, were also included in the revision of the "Suicide CARE." Among 391 suicide completers, 92.3\% $(n=361)$ presented warning signals before suicide completion, whereas $6.1 \%$ $(\mathrm{n}=24)$ did not present warning signals. In addition, $1.5 \%(\mathrm{n}=6)$ were not aware of the warning signals. In the 361 suicide completers who presented warning signs before death, alterations in verbal expression, behaviors, and emotions, $77.0 \%(\mathrm{n}=278)$ were not recognized, whereas only $20.5 \%(n=74)$ were recognized. In addition, warning sign recognitions in $2.5 \%(\mathrm{n}=9)$ was not evaluated.

The psychological autopsy interview reports classified suicide warning signals into three groups: verbal, behavioral, and situational signals. First, the verbal and behavioral signals for a total of 249 people who committed suicide from 2016 to 2018, were analyzed descriptively since the data from the suicide completers in 2015 did not contain specific contents. The specific suicidal signals were as follows: the verbal signals were classified into frequent mentions about suicide, homicide, or death $(n=130,52.2 \%)$, somatic complaints $(n=120,48.2 \%)$, expression of self-criticism $(n=106,42.6 \%)$, questioning on how to commit suicide ( $\mathrm{n}=30,12.0 \%)$, writing about death in letters, notes, etc. ( $n=40,16.1 \%)$, expression of longing for the afterlife $(\mathrm{n}=30,12.0 \%)$, and talking about people who committed suicide ( $n=19,7.6 \%)$. The behavioral signals were classified into alterations in sleep $(n=164,65.9 \%)$, alterations in appetite $(n=133,53.4 \%)$, decreased concentration or indecisiveness $(\mathrm{n}=82,32.9 \%)$, indifference to appearance management $(\mathrm{n}=82$, $32.9 \%$ ), disposing of the belongings ( $n=75,30.1 \%$ ), self-destructive behaviors or substance abuse $(n=63,25.3 \%)$, striving 
Table 2. International gatekeeper training programs for suicide prevention

\begin{tabular}{|c|c|c|}
\hline Program & Requirement & Education \\
\hline $\begin{array}{l}\text { Applied Suicide Intervention } \\
\text { Skills Training (ASIST) }{ }^{79}\end{array}$ & $\begin{array}{l}\text { Instructor education: } \$ 2,600 \text { for } 5 \text {-day course } \\
\text { Gatekeeper education: } \$ 36 \text { for } 14 \text {-hour course }\end{array}$ & $\begin{array}{l}\text { Mini-lecture, discussion, group simulation, } \\
\text { and role-play }\end{array}$ \\
\hline 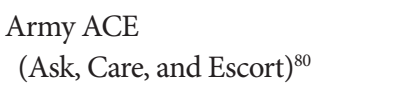 & $\begin{array}{l}\text { Gatekeeper education: peer-to-peer or } \\
\text { buddy-to-buddy education for four hours }\end{array}$ & $\begin{array}{l}\text { Encourage direct questions to peers with } \\
\text { suicidal behavior }\end{array}$ \\
\hline $\begin{array}{l}\text { At-Risk for Middle School } \\
\text { Educators }^{81}\end{array}$ & $\begin{array}{l}\text { Gatekeeper education: online education for } \\
50 \text { minutes, } \$ 5-30\end{array}$ & $\begin{array}{l}\text { Detect middle school students' psychological } \\
\text { conflicts including depressed mood and } \\
\text { suicidal ideation }\end{array}$ \\
\hline $\begin{array}{l}\text { At-Risk for High School } \\
\text { Educators }^{82}\end{array}$ & $\begin{array}{l}\text { Gatekeeper education: } \$ 500-3,500 \text { for } \\
1 \text {-year license }\end{array}$ & $\begin{array}{l}\text { Detect high school students' psychological } \\
\text { conflicts }\end{array}$ \\
\hline $\begin{array}{l}\text { At-Risk for University and } \\
\text { College Faculty }{ }^{83}\end{array}$ & $\begin{array}{l}\text { Gatekeeper education: online education for } \\
45 \text { minutes, } \$ 1,850-4,850 \text { for } 1 \text {-2-year license }\end{array}$ & $\begin{array}{l}\text { Web-based interactive simulation to analyze } \\
\text { virtual students' psychological conflicts including } \\
\text { depression, anxiety, and suicidal ideation }\end{array}$ \\
\hline $\begin{array}{l}\text { At-Risk for University and } \\
\text { College Students }^{84}\end{array}$ & $\begin{array}{l}\text { Gatekeeper education: } 30 \text {-minute course, } \\
\$ 2-20 \text { for users }\end{array}$ & $\begin{array}{l}\text { Online program to detect at-risk students, } \\
\text { discuss his or her problems, and connect with } \\
\text { a counseling center }\end{array}$ \\
\hline At-Risk in the $\mathrm{ED}^{85}$ & $\begin{array}{l}\text { Gatekeeper education: 1-hour course, } \\
\$ 35-75 \text { for users }\end{array}$ & $\begin{array}{l}\text { Online program to detect the signs of suicide } \\
\text { and substance abuse in patients }\end{array}$ \\
\hline Be A Link! $!^{86}$ & $\begin{array}{l}\text { Instructor education: } \$ 295 \text { for } 2 \text {-day course } \\
\text { Gatekeeper education: } \$ 375 \text { for } 2 \text {-hour course } \\
\text { (including instructor resource) }\end{array}$ & $\begin{array}{l}\text { Community connection and risk protocol to } \\
\text { detect risk factor and a warning sign of suicide }\end{array}$ \\
\hline Campus Connect $^{87}$ & $\begin{array}{l}\text { Instructor education: } \$ 4,500 \text { for 6-hour workshop } \\
\text { Gatekeeper education: } 2.5 \text {-hour training }\end{array}$ & $\begin{array}{l}\text { Information about risky and protective factors of } \\
\text { suicide, role-play to train communication skills }\end{array}$ \\
\hline $\begin{array}{l}\text { Connect Suicide Prevention } \\
\text { Intervention Training } \\
88\end{array}$ & $\begin{array}{l}\text { Instructor education: } \$ 6,000 \text { for } 3 \text {-day course } \\
\text { Gatekeeper education: } \$ 1,600 \text { for } 4 \text { to } 6 \text {-hour course }\end{array}$ & $\begin{array}{l}\text { Educate communication and link, decrease stigma, } \\
\text { restrict lethal tools }\end{array}$ \\
\hline $\begin{array}{l}\text { Connect Suicide Postvention } \\
\text { Training }{ }^{89}\end{array}$ & $\begin{array}{l}\text { Instructor education: } \$ 6,000 \text { for } 3 \text {-day course } \\
\text { Gatekeeper education: } \$ 1,600 \text { for } 4 \text { to 6-hour course }\end{array}$ & $\begin{array}{l}\text { Increase collaboration to provide the most } \\
\text { effective intervention, care for a survivor, } \\
\text { and connect with community society }\end{array}$ \\
\hline $\begin{array}{l}\text { Connect Youth Leaders } \\
\text { Partnering with Adults in } \\
\text { Youth Suicide Prevention }^{90}\end{array}$ & Gatekeeper education: $\$ 6,000$ for a 2-day course & Adolescent suicide prevention program \\
\hline EndingSuicide.Com ${ }^{78}$ & Gatekeeper education: two modules on the homepage & $\begin{array}{l}\text { Simple module for persons who have no health } \\
\text { education and a complex module for health } \\
\text { professionals }\end{array}$ \\
\hline $\begin{array}{l}\text { Family of Heroes: Training for } \\
\text { Family Members for Veterans }{ }^{91}\end{array}$ & $\begin{array}{l}\text { Gatekeeper education: } 1 \text {-hour course, } \\
\$ 2-2.5 \text { for users }\end{array}$ & Simulation conversation with a virtual veteran \\
\hline $\begin{array}{l}\text { Gryphon Place Gatekeeper } \\
\text { Suicide Prevention Program- } \\
\text { A Middle School Curriculum }^{92}\end{array}$ & Gatekeeper education: 3 -day course & Detect, intervene, and help adolescents at-risk \\
\hline $\begin{array}{l}\text { High School Gatekeeper } \\
\text { Curriculum }^{92}\end{array}$ & Gatekeeper education: four lessens & Detect, intervene, and help adolescents at-risk \\
\hline 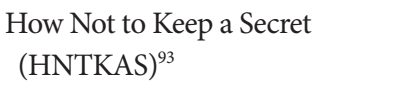 & $\begin{array}{l}\text { Gatekeeper education: } \$ 50 \text { for a one-day course } \\
\text { (including free DVD) }\end{array}$ & $\begin{array}{l}\text { Include clinical presentation, documentary, } \\
\text { and interview skills }\end{array}$ \\
\hline $\begin{array}{l}\text { Just Talk About It: Suicide } \\
\text { Prevention Toolkit }{ }^{78}\end{array}$ & $\begin{array}{l}\text { Instructor education: free for 2-hour course } \\
\text { Gatekeeper education: } 0.5 \text { to } 1.5 \text {-hour course }\end{array}$ & $\begin{array}{l}\text { Help adolescents with the signs of suicidal } \\
\text { ideation }\end{array}$ \\
\hline $\begin{array}{l}\text { In Harm's Way: } \\
\text { Law Enforcement } \\
\text { Suicide Prevention }\end{array}$ & Instructor education: free for 8 -hour course & $\begin{array}{l}\text { Detect warning signs of depression, PTSD, } \\
\text { and suicide through peer training }\end{array}$ \\
\hline $\begin{array}{l}\text { Late Life Suicide Prevention } \\
\text { Toolkit }^{95}\end{array}$ & Gatekeeper education: free & $\begin{array}{l}\text { Detect suicide warning signs, establish a relationship, } \\
\text { evaluate suicide risk and resilience factors }\end{array}$ \\
\hline
\end{tabular}


Table 2. International gatekeeper training programs for suicide prevention (continued)

\begin{tabular}{|c|c|c|}
\hline Program & Requirement & Education \\
\hline Let's Talk Gatekeeper Training ${ }^{78}$ & Gatekeeper education: 2-hour course & $\begin{array}{l}\text { Understand the risky and protective factors of } \\
\text { suicide, warning signs of suicide, and } \\
\text { communication with children and adolescents }\end{array}$ \\
\hline $\begin{array}{l}\text { Lifelines Intervention: Helping } \\
\text { Students At Risk for Suicide }{ }^{96}\end{array}$ & $\begin{array}{l}\text { Gatekeeper education: } \$ 149 \text { for manual (216 pages) } \\
\text { and DVD ( } 37 \text { minutes) }\end{array}$ & $\begin{array}{l}\text { Modify classical counseling skills about the } \\
\text { school environment }\end{array}$ \\
\hline Life Savers Training ${ }^{97}$ & Gatekeeper education: $\$ 230$ for 3-day course & $\begin{array}{l}\text { Active listening, confidentiality, and help } \\
\text { peers with a psychological problem }\end{array}$ \\
\hline $\begin{array}{l}\text { Making Educators Partners in } \\
\text { Youth Suicide Prevention }\end{array}$ & Gatekeeper education: free for five modules & Email specific questions to expert personnel \\
\hline $\begin{array}{l}\text { More Than Sad: Suicide } \\
\text { Prevention Education for } \\
\text { Teachers and Other School } \\
\text { Personnel }^{99}\end{array}$ & $\begin{array}{l}\text { Gatekeeper education: } \$ 99.99 \text { for one guidebook } \\
\text { and } 2 \text { DVDs }\end{array}$ & $\begin{array}{l}\text { Educate on the life-threatening mental disorders } \\
\text { of adolescents }\end{array}$ \\
\hline $\begin{array}{l}\text { Online Counseling and } \\
\text { Suicide Intervention Specialist } \\
(\text { OCSIS })^{100}\end{array}$ & $\begin{array}{l}\text { Gatekeeper education: } \$ 199 \text { for a volunteer, } \\
\$ 399 \text { for professional }\end{array}$ & Detect, evaluate, and relieve suicide risk \\
\hline $\begin{array}{l}\text { Operation S.A.V.E.: VA Suicide } \\
\text { Prevention Gatekeeper } \\
\text { Training }\end{array}$ & Gatekeeper education: free for 1 to 2 - hour course & Include risk factors of suicide, SAVE model \\
\hline QPR (Question, Persuade, Refer) $)^{102}$ & $\begin{array}{l}\text { Gatekeeper education: } \$ 495 \text { for } 12 \text {-hour course, } \\
\$ 395 \text { for 8-hour course }\end{array}$ & $\begin{array}{l}\text { “Chain of survival” approach to recognize } \\
\text { warning signs }\end{array}$ \\
\hline QPR for Law Enforcement ${ }^{102}$ & Gatekeeper education: $\$ 119$ for 90 -minute course & $\begin{array}{l}\text { Online education program to recognize } \\
\text { warning signs of suicide }\end{array}$ \\
\hline QPR for Nurses ${ }^{103}$ & Gatekeeper education: $\$ 139$ for 3 to 6 -hour course & Evaluate suicidal risk \\
\hline $\begin{array}{l}\text { Response: A Comprehensive } \\
\text { High School-based Suicide } \\
\text { Awareness Program }{ }^{104}\end{array}$ & $\begin{array}{l}\text { Instructor education: } \$ 375 \text { for 2-hour course of teacher } \\
\text { Gatekeeper education: } \$ 150\end{array}$ & $\begin{array}{l}\text { Detect depression and suicidal ideation, } \\
\text { and recommend services }\end{array}$ \\
\hline $\begin{array}{l}\text { Shield of Care: A System-Focused } \\
\text { Approach to Protecting Juvenile } \\
\text { Justice Youth from Suicide }{ }^{105}\end{array}$ & Gatekeeper education: free for 8-hour course & $\begin{array}{l}\text { Suicide prevention system-centered model to } \\
\text { connect with adolescents }\end{array}$ \\
\hline Sources of Strength ${ }^{106}$ & $\begin{array}{l}\text { Instructor education: } \$ 5,000 \text { for } 40 \text {-hour course } \\
\text { Gatekeeper education: } 15 \text {-hour course }\end{array}$ & $\begin{array}{l}\text { Send a message of "Hope, Help, and Strength" } \\
\text { through a presentation, poster, video, and } \\
\text { the internet }\end{array}$ \\
\hline Student Support Network ${ }^{107}$ & $\begin{array}{l}\text { Instructor education: } \$ 1,000-3,000 \text { for } 1 \text { to } 2 \text {-day course } \\
\text { Gatekeeper education: free }\end{array}$ & $\begin{array}{l}\text { Include mental and behavioral health problems } \\
\text { and knowledge about the community }\end{array}$ \\
\hline $\begin{array}{l}\text { Suicide Alertness for Everyone } \\
(\text { safeTALK) })^{108}\end{array}$ & $\begin{array}{l}\text { Instructor education: } \$ 820 \text { for 2-day workshop } \\
\text { Gatekeeper education: } \$ 6.50-7.50\end{array}$ & $\begin{array}{l}\text { A structured behavior training program with } \\
\text { graded exposure }\end{array}$ \\
\hline $\begin{array}{l}\text { Student and Aging: } \\
\text { A Gatekeeper's Workshop }{ }^{109}\end{array}$ & Gatekeeper education: 4-hour course & Detect the risk factors of suicide in the elderly \\
\hline $\begin{array}{l}\text { Suicide Prevention Training for } \\
\text { Gatekeeper of Older Adult }{ }^{110}\end{array}$ & Gatekeeper education: free for 8-hour course & $\begin{array}{l}\text { Include aging, mental health, and suicide, } \\
\text { risk and protective factors of suicide }\end{array}$ \\
\hline Trevor Lifeguard Workshop ${ }^{111}$ & $\begin{array}{l}\text { Instructor education: 2-day course } \\
\text { Gatekeeper education: 1-hour course }\end{array}$ & $\begin{array}{l}\text { Two versions, LGBTQ adolescents and ordinary } \\
\text { adolescents }\end{array}$ \\
\hline Veterans on Campus ${ }^{12}$ & Gatekeeper education: $\$ 2,500$ & $\begin{array}{l}\text { Discuss psychological conflicts of veterans and } \\
\text { connect with community resources }\end{array}$ \\
\hline $\begin{array}{l}\text { Working Minds: Suicide } \\
\text { Prevention in the Workplace }{ }^{113}\end{array}$ & Instructor education: $\$ 1,000$ for 1 -day course & $\begin{array}{l}\text { Educate on interviewing skills about } \\
\text { psychological conflicts of workers }\end{array}$ \\
\hline
\end{tabular}

Adapted from Suicide Prevention Resource Center. Resources and Programs [Internet]. Waltham, MA: Suicide Prevention Resource Center; 2020 , according to the Creative commons license ${ }^{81}$ 
to improve interpersonal relationships ( $\mathrm{n}=45,18.1 \%)$, planning suicide ( $n=43,17.3 \%)$, aggressive or impulsive behaviors $(n=43$, $17.3 \%)$, giving others the things they usually valued $(n=19$, $7.6 \%$ ), and excessive collecting of poems, music, and movies related to death $(\mathrm{n}=12,4.8 \%)$. Second, the situational signals for a total of 103 people who completed suicides in 2018 only were analyzed descriptively. Since situational signals for suicide can be multifactorial and complex, they were simply classified as mental health problems $(\mathrm{n}=87,84.5 \%)$, occupation-related stress $(n=70,68.0 \%)$, economic problems $(n=56,54.4 \%)$, family-related stress $(n=56,54.4 \%)$, interpersonal relationship-related stress $(n=40,38.8 \%)$, spouse-related stress $(n=35,34.0 \%)$, physical health problems $(n=34,33.0 \%)$, lover-related stress $(\mathrm{n}=14,13.6 \%)$, and learning-related stress $(\mathrm{n}=14,13.6 \%)$. In terms of classification of the suicide commitment period, the warning signals three months before the suicide completion were alterations in emotion, alterations in appetite, alterations in sleep, disposing of the belongings, and loss of energy or interest. In addition, the warning signals one week before suicide completion were disposing of belongings, striving to improve interpersonal relationships, and aggressive or impulsive behaviors. Furthermore, in terms of classification of the life cycle, the suicide warning signals of young adults were learning, family and lover-related stress, loneliness, and absence of close relationships. In addition, the signals in middle-aged adults were economic stress and debt problems. Finally, the signals in the elderly were chronic physical diseases, unspecified somatic symptoms, and absence of interpersonal relationships.

As shown in Figure 2, based on the psychological autopsy interview reports, the chronicled pathways to suicide completion of job seekers, self-employed persons, and retirees were conceptualized to improve the understanding of suicidal cases. Thus, by consensus of the developers for "Suicide CARE" version 2.0, life cycle-based example cases were selected from the conceptualized paths to suicide.

\section{Feedback of persons who completed "Suicide CARE" version 1.6}

From September 16 to October 4, 2019, a survey question- naire that included difficulties in teaching, feedback about content and construction of each part, and feedback about instructor training courses was administered by persons who completed training of "Suicide CARE" version 1.6, and 66 persons responded to the survey questionnaire. ${ }^{82}$ The responses were as follows: First, since many of the students for "Suicide CARE" version 1.6 felt bored, it has been proposed that a method to relieve boredom should be added in the new version. Thus, "Suicide CARE" version 2.0 is needed to fulfill a diversified demand for education according to age, sex, occupation, and other factors. In addition, the transcript of the lecture needs to be reduced, and the capability of educators needs to be increased in order to guarantee educators' unconstrained lectures. Furthermore, the education time needs to be reduced to decrease the burden of educators and students. It has been proposed that weighting more important content can improve the efficiency of lectures. Second, it has been proposed that the contents of the adolescent version should be updated and added. Most of all, it is necessary to add the fact that suicide currently is the most common cause of death in adolescence. It is also necessary to add the fact that self-injury is a warning sign for suicide. Moreover, it is necessary to complement a method to improve the concentration of middle school students. Third, it has been proposed that "Suicide CARE" version 2.0, including the transcript of a lecture, should be differentiated according to the life cycle or occupation (i.e., adolescents, elders, office workers, public servants, soldiers, teachers, college students, etc.). It has been proposed that an advanced course should be developed in "Suicide CARE" version 2.0. In addition, it is necessary to diversify the scenarios of role-playing to improve the quality of the practical exercise for educators. Overall, the contents of the survey responses were consistent with the opinions of the developers for "Suicide CARE" version 2.0.

\section{DISCUSSION}

The developers of "Suicide CARE" version 2.0 planned to revise more than $70 \%$ of "Suicide CARE" version 1.6 based

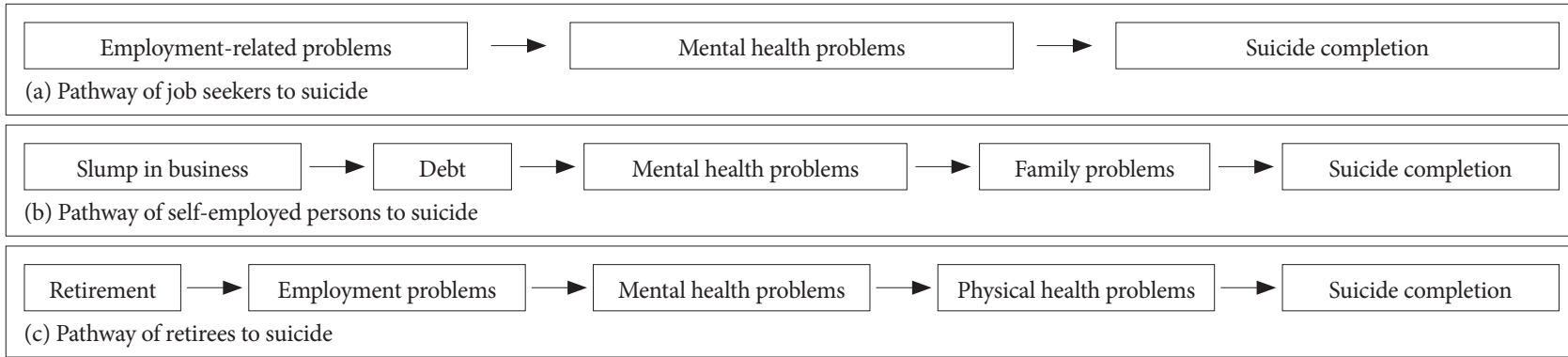

Figure 2. Pathways to suicide completion. These pathways were proposed following the psychological autopsy interview reports collected between 2015 and 2018. 
Table 3. Comparison of "Suicide CARE" versions 1.6 and 2.0

\begin{tabular}{|c|c|c|}
\hline & Version 1.6 & Version 2.0 \\
\hline Introduction & - Introduce suicide epidemiological data & $\begin{array}{l}\text { - Quote the psychological autopsy interview reports (2015-2018) } \\
\text { - Emphasize the importance of the gatekeeper training program } \\
\text { - Quote written texts authored by Prof. Se-Won Lim }\end{array}$ \\
\hline "Careful observation" & $\begin{array}{l}\text { - Introduce theoretically relevant verbal, } \\
\text { behavioral, and situational warning signs } \\
\text { of suicide completers }\end{array}$ & $\begin{array}{l}\text { - Introduce the real verbal, behavioral, and situational warning signs of } \\
\text { suicide completers, based on the psychological autopsy interview } \\
\text { reports (2015-2018) } \\
\text { - Explain the characteristics of the warning signs according to life cycles }\end{array}$ \\
\hline "Active listening" & $\begin{array}{l}\text { - Ask a question about suicidal ideation } \\
\text { - Explain the concept of ambivalence }\end{array}$ & $\begin{array}{l}\text { - Connect the warning signs of "Care observation" with the question of } \\
\text { "Active listening" } \\
\text { - Change the explaining pattern and design about the concept of ambivalence }\end{array}$ \\
\hline $\begin{array}{l}\text { "Risk evaluation and } \\
\text { Expert referral" }\end{array}$ & - Lists for safety check & $\begin{array}{l}\text { - Dichotomize the lists for safety into the evaluation of suicide risk } \\
\text { and helping safely } \\
\text { - Change video clips and role play }\end{array}$ \\
\hline
\end{tabular}

on the troubling suicide-related situation in Korea. Psychological autopsy interview reports showed that suicide completers express self-deprecation and show changes in sleep and appetite. Thus, the findings of the psychological autopsy interview reports were planned to be incorporated into video clip scenarios. It has also been planned that the characters of video clips include a job-seeker (aged 20-30, female), a selfemployed person (middle aged, male), and a retired person (over 70 years old, male). Herein, from August 2019 to February 2020, the workbook, manuscript of lecture, and video clips of "Suicide CARE" were revised. Since "Suicide CARE" version 2.0 was developed based on extensive international literature reviews and the psychological characteristics of more than 300,000 Koreans, it is expected to enhance the effectiveness of gatekeeper training. In "Suicide CARE" version 2.0, educational video clips are regarded as an important component because it is a key medium that delivers the main contents of education to the trainee. Herein, as shown in Table 3, "Suicide CARE" has been updated from version 1.6 to 2.0. ${ }^{114}$ In terms of a utilization plan, from now on it is expected that all gatekeeper education programs be conducted using "Suicide CARE" version 2.0. Moreover, it is expected that "Suicide CARE" version 2.0 be positioned as the basic framework for many developing gatekeeper training programs for suicide prevention in Korea in the near future. However, owing to the limited production cost, there has been a limit on capturing visual quality that can convey empathy and emotion along with educational content. In the future, when revising "Suicide CARE" version 2.0 or when creating a specialized program for other special roles, such as firefighters and soldiers, it is necessary to reflect sufficient production costs in the production of the video when forecasting a budget.

\section{Acknowledgments}

We would like to express our sincere gratitude to Prof. Se-Won Lim for his dedication to the development of "Suicide CARE" versions 1.0 and 1.6. This work was supported by the Standardized Suicide Prevention Program for Gatekeeper Intervention in Korea, version 2.0, development grant from the Korea Suicide Prevention Center. This work was also supported by the Korea Psychological Autopsy Center.

\section{Conflicts of Interest}

The authors have no potential conflicts of interest to disclose.

\section{Author Contributions}

Conceptualization: Hwa-Yong Lee, Hong Jin Jeon, Jong Woo Paik, Kang Seob Oh. Funding acquisition: Hwa-Yong Lee. Investigation: all authors. Projected administration: all authors. Writing-original draft: Seon-Cheol Park, Kyoung-Sae Na, Hwa-Young Lee. Writing_review \& editing: all authors.

\section{ORCID iDs}

Seon-Cheol Park

Kyoung-Sae Na

Sun-Jung Kwon

Minjae Kim

Hyoung-Jun Kim

Myungjae Baik

Jinmi Seol

Eun Ji An

Sang Min Lee

Eun-Jin Lee

Meerae Lim

Sung Joon Cho

Gwang Hun Kim

Nari Kim

Hong Jin Jeon

Jong-Woo Paik

Kang Seob Oh

Hwa-Young Lee https://orcid.org/0000-0003-3691-4624 https://orcid.org/0000-0002-0148-9827 https://orcid.org/0000-0001-9938-2237 https://orcid.org/0000-0003-4777-7397 https://orcid.org/0000-0003-0037-4726 https://orcid.org/0000-0001-8989-7405 https://orcid.org/0000-0001-9734-7243 https://orcid.org/0000-0001-6204-9481 https://orcid.org/0000-0002-7834-8272 https://orcid.org/0000-0002-1579-1007 https://orcid.org/0000-0001-7504-7485 https://orcid.org/0000-0001-6981-0931 https://orcid.org/0000-0002-8407-2663 https://orcid.org/0000-0003-2183-3645 https://orcid.org/0000-0002-6126-542X https://orcid.org/0000-0002-1804-8497 https://orcid.org/0000-0001-9850-1898 https://orcid.org/0000-0002-2749-6232

\section{REFERENCES}

1. Han C. How to approach to suicide prevention. J Korean Med Assoc 2019;62:79-84.

2. Kim S, Lee KU. Neurobiological understanding of suicide. J Korean Med Assoc 2019;62:85-92. 
3. Kim SW, Jhon M, Kim M, Paik JW, Kim JM, Yoon JS. A social psychiatric approach to suicide prevention. J Korean Med Assoc 2019;62:93101.

4. Lee SU, Park JI. Medical policy for suicide prevention. J Korean Med Assoc 2019;62:102-106.

5. Organization for Economic Cooperation and Development (OECD). OECD Data: Suicidal rates [Internet]. Available at: https://data.oecd. org/healthstat/suicide-rates.htm. Accessed April 30, 2020.

6. Ministry of Health and Welfare. National Suicide Prevention Action Plan [Internet]. Sejong. Available at: http://www.mohw.go.kr/react/ a l/s a $10301 \mathrm{vw}$.j sp? PAR_MEN U_ ID = $04 \& \mathrm{MENU}_{-}$ $\mathrm{ID}=0403$ \&page $=44 \&$ CONT_SEQ=343649. Accessed April 30, 2020.

7. Zalsman G, Hawton K, Wasserman D, van Heeringen K, Arensman E, Sarchiapone M, et al. Suicide prevention strategies revisited: 10year systematic review. Lancet Psychiatry 2016;3:649-659.

8. Centers for Disease Control and Prevention. Youth Suicide Prevention Programs: A Resource Guide. Atlanta, GA: Centers for Disease Control and Prevention; 1992.

9. Korea Association for Suicide Prevention. "Suicide CARE” (Standardized Suicide Prevention Program for Gatekeeper Intervention in Korea) version 1.0. Seoul: Korea Association for Suicide Prevention; 2011.

10. Paik JW, Jo SJ, Lee S, Ong JY, Park JI. The effect of Korean standardized suicide prevention program on intervention by gatekeepers. J Korean Neuropsychiatr Assoc 2014;53:358-363.

11. Korea Association for Suicide Prevention \& Korea Suicide Prevention Center. "Suicide CARE" (Standardized Suicide Prevention Program for Gatekeeper Intervention in Korea) version 1.6. Seoul: Korea Association for Suicide Prevention\& Korea Suicide Prevention Center; 2011.

12. Korea Psychological Autopsy Center. Psychological Autopsy Interview Report 2015-2018. Seoul: Korea Psychological Autopsy Center; 2018.

13. Korea Suicide Prevention Center. Registration System for Domestic Gatekeeper Training Programs to Introduce Evidence-Based Suicide Prevention Interventions [Internet]. Seoul. Available at: http://www. spckorea.or.kr/index.php. Accessed April 30, 2020.

14. Korea Suicide Prevention Center. Suicide Reporting Recommendation Guideline 3.0. Seoul: Korea Suicide Prevention Center; 2014.

15. Korea Suicide Prevention Center. Suicide Prevention Education Program for Senior Service Provider. Seoul: Korea Suicide Prevention Center; 2015.

16. Korea Suicide Prevention Center. Cognitive Behavioral Program for Suicidal Attempter. Seoul: Korea Suicide Prevention Center; 2015.

17. Korea Suicide Prevention Center. 'Suicide CARE' for Air Force. Seoul: Korea Suicide Prevention Center; 2017.

18. Korea Suicide Prevention Center. Senior Suicide Prevention Education Program. Seoul: Korea Suicide Prevention Center; 2017.

19. Korea Suicide Prevention Center. Suicide Prevention Education Program for Office Worker. Seoul: Korea Suicide Prevention Center; 2017.

20. Korea Suicide Prevention Center. Suicide Prevention Education for Emergency Medical Worker. Seoul: Korea Suicide Prevention Center; 2018.

21. Korea Suicide Prevention Center. Suicide Prevention Gatekeeper Education Program. Seoul: Korea Suicide Prevention Center; 2018.

22. Korea Suicide Prevention Center. Senior Suicide Prevention Gatekeeper Education Program. Seoul: Korea Suicide Prevention Center; 2018.

23. Seoul Suicide Prevention Center. Crisis Management Protocol for Suicide and Mental Disorder. Seoul: Seoul Suicide Prevention Center; 2014.

24. Seoul Suicide Prevention Center. Suicide Crisis Intervention Handbook. Seoul: Seoul Suicide Prevention Center; 2014.

25. Seoul Suicide Prevention Center. Suicide Prevention Gatekeeper
Training Program. Seoul: Seoul Suicide Prevention Center; 2014.

26. Seoul Suicide Prevention Center. Manual for Counseling and SelfHelp Group for Survivor of Bereavement by Suicide. Seoul: Seoul Suicide Prevention Center; 2015.

27. Seoul Suicide Prevention Center. Suicide Prevention Education for Emergency Worker. Seoul: Seoul Suicide Prevention Center; 2017.

28. Seoul Suicide Prevention Center. Suicide Prevention Professional Training Program. Seoul: Seoul Suicide Prevention Center; 2017.

29. Seoul Suicide Prevention Center. Gatekeeper Training Program for Teacher. Seoul: Seoul Suicide Prevention Center; 2018.

30. Incheon Suicide Prevention Center. Adolescent Suicide Crisis Intervention Manual for Teacher and Official. Incheon: Incheon Suicide Prevention Center; 2015.

31. Seoul Mental Health Welfare Center. School-Based Suicide Postvention Program. Seoul: Seoul Mental Health Welfare Center; 2015.

32. Gangseo-gu Mental Health Welfare Center. Suicide Prevention Program of Community Resident Participation. Seoul; Gangseo-gu Mental Health Welfare Center; 2015.

33. Seongdong-gu Mental Health Welfare Center. Mental Health Promotion and Happiness Enhancement Thank You Program. Seoul: Seongdong-gu Mental Health Welfare Center; 2014.

34. Busan Mental Health Welfare Center. 'Mind Cane.' Busan: Busan Mental Health Welfare Center; 2017.

35. Busan Mental Health Welfare Center. Psychological Emotion Support Recommendation for Official Whose Client Commit Suicide. Busan: Busan Mental Health Welfare Center; 2018.

36. Incheon Mental Health Welfare Center. Psychological Support for Survivor of Bereavement by Suicide. Incheon: Incheon Mental Health Welfare Center; 2015.

37. Incheon Mental Health Welfare Center. Senior Suicidal Crisis Management Manual. Incheon: Incheon Mental Health Welfare Center; 2016.

38. Incheon Mental Health Welfare Center. 'Suicide Prevention and Crisis Intervention Manual' for Suicide Prevention Official. Incheon: Incheon Mental Health Welfare Center; 2017.

39. Daegu Mental Health Welfare Center. Adolescent Life Respect Education. Daegu: Daegu Mental Health Welfare Center; 2019.

40. Daegu Mental Health Welfare Center. Senior Life Respect Education Program, 'Life Charger.' Daegu: Daegu Mental Health Welfare Center; 2019.

41. Gwangju Mental Health Welfare Center. Crisis Intervention Program of Gwangju. Gwangju: Gwangju Mental Health Welfare Center; 2016.

42. Buk-gu Mental Health Welfare Center. 'Life Zone.' Gwangju: Buk-gu Mental Health Welfare Center; 2018.

43. Buk-gu Mental Health Welfare Center. 'Mind Friends.' Gwangju: Buk-gu Mental Health Welfare Center; 2019.

44. Dong-gu Mental Health Welfare Center. 'What's Up?' Gwangju: Dong-gu Mental Health Welfare Center; 2018.

45. Gyeonggi-do Mental Health Welfare Center. Group Program Manual for Survivor of Bereavement by Suicide. Suwon: Gyeonggi-do Mental Health Welfare Center; 2017.

46. Gyeonggi-do Mental Health Welfare Center. Life Loving Teen Teen Class 3.0. Suwon: Gyeonggi-do Mental Health Welfare Center; 2017.

47. Gyeonggi-do Mental Health Welfare Center. Life Loving Teen Teen Class Gatekeeper. Suwon: Gyeonggi-do Mental Health Welfare Center; 2019.

48. Gyeonggi-do Mental Health Welfare Center. Student Suicide Prevention Intervention Program, 'M-love'. Suwon: Gyeonggi-do Mental Health Welfare Center; 2019.

49. Suwon Mental Health Welfare Center. Gold Medal Case Management Program. Suwon: Suwon Mental Health Welfare Center; 2017.

50. Suwon Mental Health Welfare Center. Adolescent Suicide Prevention Gatekeeper Training Program, 'Between Friends.' Suwon: Suwon Mental Health Welfare Center; 2017. 
51. Seongnam Mental Health Welfare Center. Life Respect Manual. Seongnam: Seongnam Mental Health Welfare Center; 2014.

52. Seongnam Mental Health Welfare Center. Suicide Prevention Gatekeeper Education Program. Seongnam: Seongnam Mental Health Welfare Center; 2014.

53. Seongnam Mental Health Welfare Center. Support Guidebook for Survivor of Bereavement by Suicide. Seongnam: Seongnam Mental Health Welfare Center; 2015.

54. Hwaseong Mental Health Welfare Center Life Respect. Green Village. Hwaseong: Hwaseong Mental Health Welfare Center; 2014.

55. Chuungcheongbuk-do Mental Health Welfare Center. Gatekeeper Training Program for Police Officer. Cheongju: Chuungcheongbukdo Mental Health Welfare Center; 2018.

56. Chungcheongbuk-do Mental Health Welfare Center. Gatekeeper Training Program for Firefighter. Cheongju: Chungcheongbuk-do Mental Health Welfare Center; 2018.

57. Chungcheongbuk-do Mental Health Welfare Center. Emergency Kit Emergency Box. Cheongju: Chungcheongbuk-do Mental Health Welfare Center; 2014.

58. Chungcheongbuk-do Mental Health Welfare Center. 'Suicide Crisis Management Manual.' Cheongju: Chungcheongbuk-do Mental Health Welfare Center; 2015.

59. Chungcheongbuk-do Mental Health Welfare Center. Life Savor Training Program. Cheongju: Chungcheongbuk-do Mental Health Welfare Center; 2015.

60. Chungcheongbuk-do Mental Health Welfare Center. Chungbuk SOS (Stop of Suicide) Project. Cheongju: Chungcheongbuk-do Mental Health Welfare Center; 2017.

61. Chungcheongnam-do Mental Health Welfare Center. Running Life. Hongseong: Chungcheongnam-do Mental Health Welfare Center; 2017.

62. Gumi Mental Health Welfare Center. Motivation Enhancement Cognitive Behavioral Program 2.0, 'Empathy 3.' Gumi: Gumi Mental Health Welfare Center; 2016.

63. Gumi Mental Health Welfare Center. Short-term Intervention Program 2.0, 'Empathy 1'Gumi: Gumi Mental Health Welfare Center; 2016.

64. Gumi Mental Health Welfare Center. Peer Life Savor Training Program Gumi: Gumi Mental Health Welfare Center; 2016.

65. Jeollabuk-do Mental Health Welfare Center. Life Loving Gatekeeper Training Program and Instructor Training Program. Jeonju: Jeollabuk-do Mental Health Welfare Center; 2016.

66. Jeollabuk-do Mental Health Welfare Center. Suicide Prevention Program. Jeonju: Jeollabuk-do Mental Health Welfare Center; 2018.

67. Jeollabuk-do Mental Health Welfare Center. Life Loving Gatekeeper Professional Instructor Program. Jeonju: Jeollabuk-do Mental Health Welfare Center; 2019.

68. Wanju Mental Health Welfare Center. 'Knocking Repeatedly' Wanju: Wanju Mental Health Welfare Center; 2019.

69. Jeollanam-do Mental Health Welfare Center. 'Hello?' Naju: Jeollanam-do Mental Health Welfare Center; 2017.

70. Jeju-do Mental Health Welfare Center. Life Loving Gatekeeper Training Program. Jeju: Jeju-do Mental Health Welfare Center; 2018.

71. Korea National Police Agency. Gatekeeper Education Program for Police Officer. Seoul: Korea National Police Agency; 2018.

72. The Catholic University of Korea. Suicidality Screening Program. Seoul: The Catholic University of Korea; 2016.

73. Nazarene University. TLC Academy, 'Life Loving.' Cheonan: Nazarene University; 2015.

74. Life Respect Education Association. Life Saver Education Book Life. Seoul: Respect Education Association; 2016.

75. Life Line Korea, Samsung Life Insurance, Community Chest of Korea, \& Korea Health Promotion Institute. Adolescent Suicide Prevention Education Program, 'I Love You.' Seoul: Life Line Korea, Samsung Life Insurance, Community Chest of Korea, \& Korea Health
Promotion Institute; 2016.

76. Life Hope Protestant Suicide Prevention Center. Life Loving Gatekeeper Basic Education Program. Seoul: Life Hope Protestant Suicide Prevention Center: 2017.

77. Korean Gay Humanity Movement Organization. Sexual Minority Suicide Prevention Gatekeeper Training Program. Seoul: Korean Gay Humanity Movement Organization; 2019.

78. Suicide Prevention Resource Center. Resources and Programs [Internet]. Waltham, MA: Suicide Prevention Resource Center; 2020. Available at: http:// http://www.sprc.org/resources-programs. Accessed October 30, 2019.

79. Livingworks. Applied Suicide Intervention Skills Training (ASIST). Available at: https://www.livingworks.net/asist. Accessed April 29, 2020.

80. U.S.Army. Ask-Care-Escort: suicide intervention facilitator's handbook version 1.7. https://umtclasp.com/wp-content/ uploads/2019/01/4-Hour_ACE-SI_FacilitatorHandbook-v1-7_5Jun-17.pdf.

81. Kognito Interactive. At-Risk for Middle School Educators. Available at: https://kognito.com/products/at-risk-for-middle-school-educators. Accessed April 29, 2020.

82. Kognito Interactive. At-Risk for High School Educators. Available at: https://kognito.com/products/at-risk-for-high-school-educators. Accessed April 29, 2020.

83. Kognito Interactive. At-Risk for Faculty \& Staff. Available at: https:// store.kognito.com/products/at-risk-for-university-and-college-faculty. Accessed April 29, 2020.

84. Kognito Interactive. At-Risk for University \& College Students. Available at: https://go.kognito.com/rs/143-HCJ-270/images/HiEd_AtRisk_Student_followupstudy.pdf. Accessed April 29, 2020.

85. Albright G, Bryan C, Adam C, McMillan J, Shockley K. Using virtual patient simulations to prepare primary health care professionals to conduct substance use and mental health screening and brief intervention. J Am Psychiatr Nurses Assoc 2018;24:247-259.

86. Yellow Ribbon. Training Programs: Adult Gate Keeper Training. Available at: https://yellowribbon.org/what-we-do/training-programs/adult-gatekeeper-training.html. Accessed April 29, 2020.

87. Drum DJ, Brownson C, Burton Denmark A, Smith SE. New data on the nature of suicidal crises in college students: Shifting the paradigm. Prof Psychol Res Pract 2009;40:213-222.

88. NAMI New Hampshire. Suicide prevention and intervention evaluation summary. Concord, NH: NAMI New Hampshire; 2019.

89. NAMI New Hampshire. Suicide prevention evaluation summary. Concord, NH: NAMI New Hampshire; 2019.

90. Chung-Do JJ, Goebert DA, Bifulco K, Sugimoto-Matsuda J, Balberde-Kamali'i J, Ka’ae D, et al. Insights in public health: safe messaging for youth-led suicide prevention awareness: examples from Hawai' i. Hawaii J Med Public Health 2016;75:144-147.

91. Albright G, Goldman R, Shockley KM, McDevitt F, Akabas S. Using an avatar-based simulation to train families to motivate veterans with post-deployment stress to seek help at the VA. Games Health J 2012;1:21-28.

92. Gryphon place. Suicide Prevention \& Crisis Support. Available at: https://gryphon.org/services/suicide-prevention-crisis-support. Accessed April 29, 2020.

93. Green BJ. How Not to Keep a Secret. Available at: https://www.adolescentwellness.org/wp-content/uploads/2011/06/How-Not-toKeep-A-Secret-BPR-listing.pdf. Accessed April 29, 2020.

94. St. Petersburg College. In harm's way: law enforcement suicide prevention. Available at: https://policesuicide.spcollege.edu/crisisIHW. htm. Accessed April 29, 2020.

95. Canadian Coalition for Seniors' Mental Health. Suicide Assessment \& Prevention for Older Adults: Life Saving Tools for Health Care Providers. Available at: https://ccsmh.ca/wp-content/uploads/2016/03/FacilitatorsGuide.pdf. Accessed April 29, 2020. 
96. Underwood M, Springer J, Scott MA-R. Lifelines Intervention Helping Students at Risk for Suicide. MN: Center City; 2011.

97. LifeSavers. LifeSavers. Available at: https://www.lifesaverstrainingcorp.org/. Accessed April 209, 2020.

98. The Society for the Prevention of Teen Suicide. Making educators partners in youth suicide prevention: act on facts. Available at: http:// www.sptsusa.org/educators/meptrailer/. Accessed April 29, 2020.

99. American Foundation for Suicide Prevention. More Than Sad: Suicide Prevention Education for Teachers and Other School Personnel. Available at: https://afsp.org/more-than-sad. Accessed May 3, 2020.

100. QPR Institute I. Online Counseling and Suicide Intervention Specialist (OCSIS). Available at: https://courses.qprinstitute.com/index. php?option=com_zoo\&task=item\&item_id=3\&Itemid=101. Accessed May 3, 2020.

101. Waliski A, Matthieu M M, Townsend J.C, Castro M, Kirchner J.?Training as a recruitment strategy for building military and community partnerships with faith-based organizations: the partners in care program. J Mil Gov Couns 2016;4:2-11.

102. QPR Institute I. QPR (Question, Persuade, Refer) for Law Enforcement. Available at: https://courses.qprinstitute.com/index. php?option=com_zoo\&task=item\&item_id=12\&Itemid=101. Accessed May 3, 2020.

103. QPR Institute I. QPR for Nurses. Available at: https://courses.qprinstitute.com/index.php?option=com_zoo\&task=item\&item_ id=13\&Itemid=101. Accessed May 3, 2020.

104. ColumbiaCare Services I. Response: A Comprehensive High Schoolbased Suicide Awareness Program. Available at: http://www.columbiacare.org/response.html. Accessed May 3, 2020.

105. Tennessee Department of Mental Health. Shield of Care: A SystemFocused Approach to Protecting Juvenile Justice Youth from Suicide.
Nashville, TN; Tennessee Department of Mental Health; 2012.

106. Wyman PA, Brown CH, LoMurray M, Schmeelk-Cone K, Petrova M, $\mathrm{Yu} \mathrm{Q}$, et al. An outcome evaluation of the Sources of Strength suicide prevention program delivered by adolescent peer leaders in high schools. Am J Public Health 2010;100:1653-1061.

107. Worcester Polytechnic Institute Counseling Center. The Student Support Network Training Manual. Available at: https://www.wpi.edu/ Admin/SDCC/index.html. Accessed April 29, 2020.

108. LivingWorks. Suicide Alertness for Everyone (safeTALK). Available at: https://www.livingworks.net/safetalk. Accessed May 3, 2020.

109. Family Services of the Merrimack Valley. Student and Aging: A Gatekeeper's Workshop. Available at: https://www.fsmv.org/suicide-prevention/. Accessed May 3, 2020.

110. Family Services of the Merrimack Valley. Suicide Prevention Training for Gatekeepers of Older Adults. Available at: https://www.fsmv. org/suicide-prevention/trainings. Accessed April 29, 2020.

111. The Trevor Project. Trevor Lifeguard Workshop. Available at: https:// www.thetrevorproject.org/education/lifeguard-workshop/. Accessed May 3, 2020.

112. Cate CA, Albright G. Supporting Student Veterans: utilizing gamebased role plays with virtual humans to build military cultural competency and helping behaviors in faculty and staff. OLJ 2015;19:1-17.

113. Helen and Arthur E. Johnson Depression Center. Working Minds: Suicide Prevention in the Workplace. Available at: https://www.coloradodepressioncenter.org/workingminds/. Accessed May 3, 2020.

114. SoonChunHyang Medical Industry University Cooperation Foundation. A Study for Development of Korean Standardized Suicide Prevention Program on Intervention by Gatekeepers 2.0. Asan: SoonChunHyang Medical Industry University Cooperation Foundation; 2020 . 